\title{
NEW ANTIARRHYTHMIC IN INTRAMYOCARDIAL ARTERIES' REMODELLING IN HEART STRUCTUTAL DISEASE
}

\author{
Martín-Oropesa R. ${ }^{1}$, Pazó-Sayós L. ${ }^{1}$, Delgado-Martos M.J. ${ }^{2}$, Muñoz D. ${ }^{3}$, Delgado-Baeza E. ${ }^{2}$, Quintana- \\ Villamandos B. ${ }^{1}$
}

${ }^{1}$ Hospital General Universitario Gregorio Marañón, Dept of Anaesthesiology, Madrid, Spain, 2 Instituto de Investigación Sanitaria Gregorio Marañón, liSGM, Madrid, Spain, 3 Universidad Autónoma de Madrid, Facultad de Medicina, Madrid, Spain

\section{Background and Goal of Study:}

Dronedarone is a multichannel

blocker used to treat atrial fibrillation (AF). Left hypertrophy is widely known as AF substrate.

The aim of our study was to show the effects of dronedarone

on intramyocardial arteries structure in an experimental model of arterial hypertension

and compensated left hypertrophy.

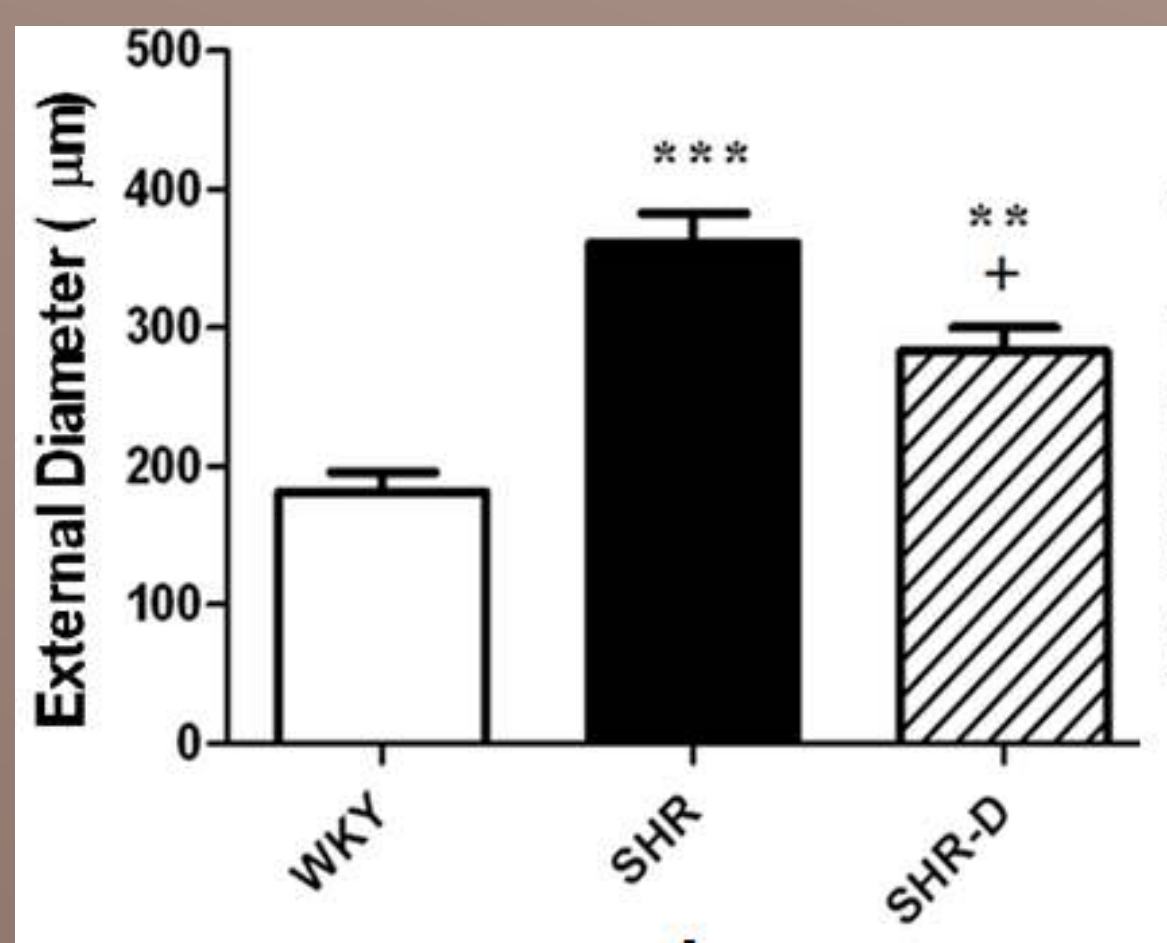

A

\section{Materials and methods:}

Adult male spontaneously hypertensive rats (SHR) were randomly divided into therapy group (SHR-D, $n=7$ ) and placebo group (SHR, $n=7$ ). Kyoto rats were used as normotensive controls (WKY, $n=7)$. After 14 days of treatment,

histological sections were prepared by staining with orcein to study intramyocardial branch of obtuse marginal artery (circumflex branch). The following variables were studied: external diameter (ED), wall width (WW), cross sectional area (CSA) and collagen volume density (CV). Comparisons among groups were made by ANOVA test of one factor with Bonferroni's correction. All data were expressed as mean \pm SEM. $\mathrm{P}<0.05$ was considered significant. All procedures were approved by the Ethics Committee of Hospital General Universitario Gregorio Marañón, Madrid, Spain.

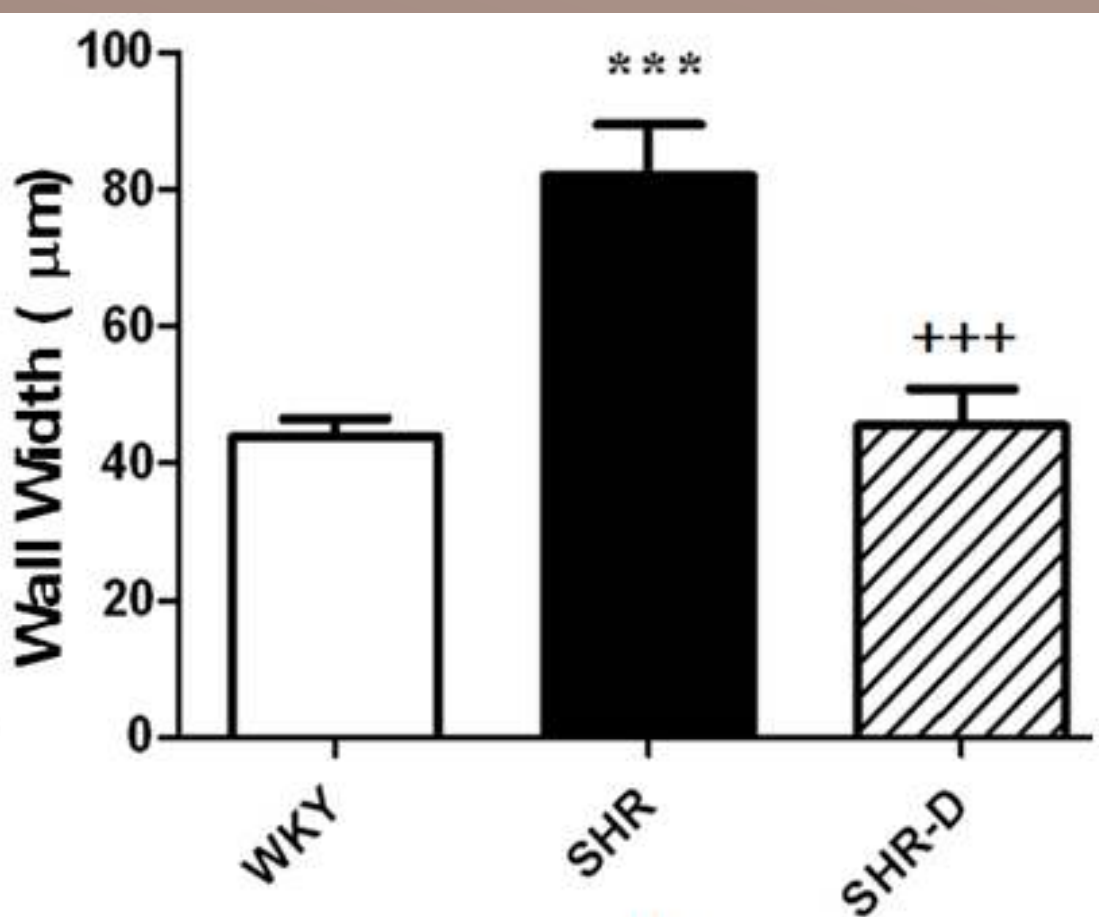

B

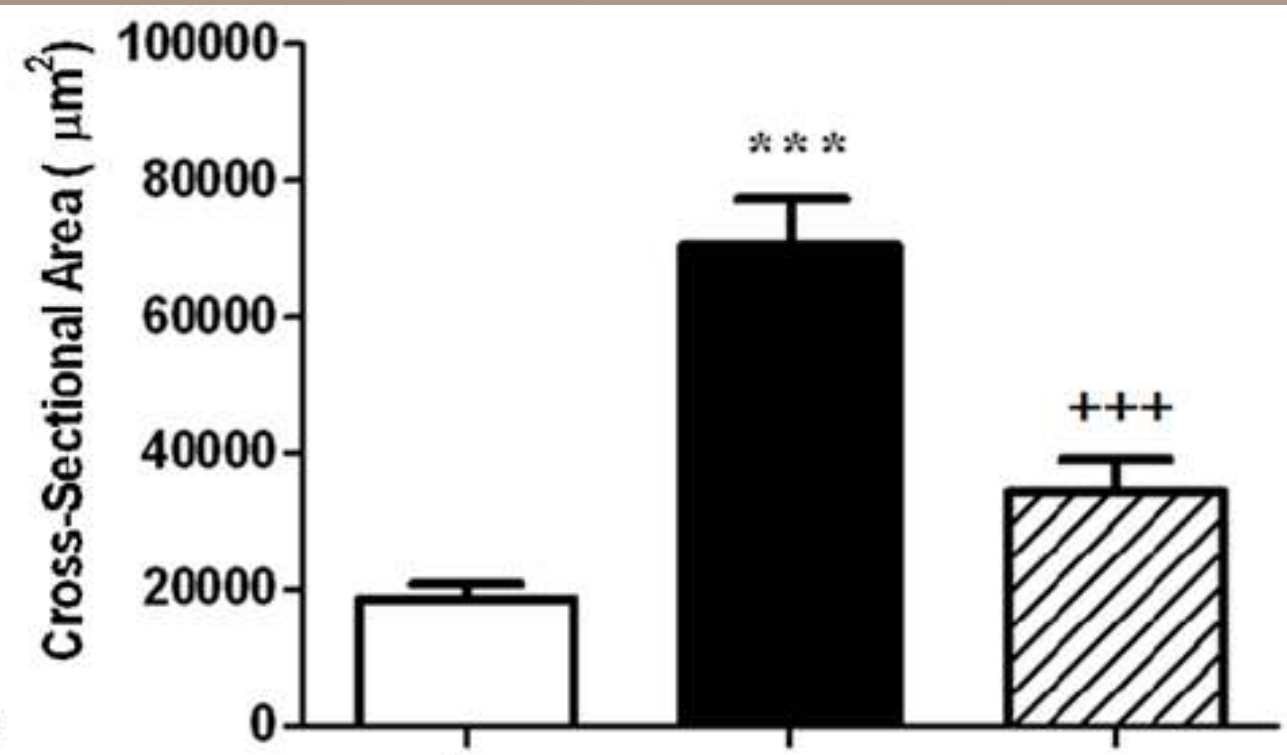

st

Fig.1 Histological characteristics of intramyocardial arteries from Wistar Kyoto control rats (WKY), spontaneously hypertensive control rats (SHR), and spontaneously hypertensive rats treated with dronedarone (SHR-D). External diameter (A), wall with (B) and cross-sectional area (C). Data are expressed as mean \pm SEM. Statistically significant differences among WKY, SHR, and SHR-D are shown: $* * P<0.01$ versus WKY; $* * * P<0.001$ versus WKY; $+P<0.05$ versus SHR; $+++P<0,001$ versus SHR
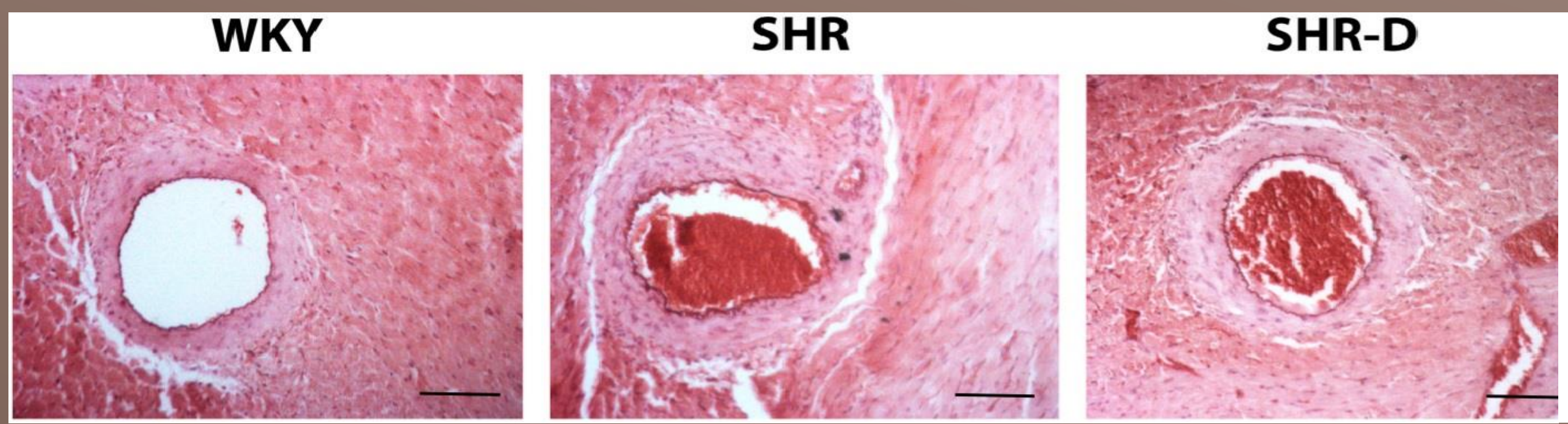

Histological sections (10x) prepared by staining with orcein to study intramyocardial branch of obtuse marginal artery from

Wistar Kyoto control rats (WKY), spontaneously hypertensive control rats (SHR), and spontaneously hypertensive rats treated with dronedarone (SHR-D).

\section{Conclusion:}

14 days of treatment with dronedarone produced a regression in intramyocardial arteries structural remodeling of spontaneously hypertensive rats. 